\title{
Las políticas y programas de alimentación y nutrición en Colombia bajo la lógica del mercado
}

\author{
Food and Nutrition Policies and Programs under the Colombian \\ market's logic
}

Lorena Patricia Mancilla López (https: //orcid.org/0000-0001-8090-8497) ${ }^{1}$ Carlos Enrique Yepes Delgado (https: //orcid.org/0000-0001-5656-4989) ${ }^{2}$

Gloria Molina Marín (https: //orcid.org/0000-0002-1035-5847) ${ }^{3}$
${ }^{1}$ Escuela de Nutrición y Dietética, Universidad de Antioquia (UdeA). Calle 70 No 52-21, Apartado Aéreo. 1226 Antioquia Colômbia. lorena.mancilla@

udea.edu.co

${ }^{2}$ Departamento de

Medicina Preventiva y

Salud Pública, Facultad de

Medicina, UdeA. Antioquia

Colômbia.

${ }^{3}$ Facultad Nacional de Salud

Pública, UdeA. Antioquia

Colômbia.

\begin{abstract}
This paper aims to understand the meanings of food and nutrition policies for beneficiary mothers and for technicians designing and implementing said policies. The Grounded Theory method was employed. The data collection techniques adopted were the semi-structured interview and the focus group. The participants were beneficiaries of food and nutrition programs, with a minimum two-year experience, and technicians with five-year experience in designing or implementing these policies and programs. Food and nutrition policies are conceived and managed under a market logic, which has required an institutional framework that adopted this commercial model to provide public services, which is implemented in the scheme of outsourcing third parties, especially private companies, who implement these policies to achieve financial profitability. The market is imposed as the benchmark for the State's actions, and, therefore, the State's action is limited the oversight of outsourced actions, and food policies become devices for the diversion of public resources to the private sector.
\end{abstract}

Key words State, Food and nutrition programs and policies, Nutrition in public health, Food assistance, Outsourced services
Resumen El propósito de este artículo es comprender los significados de las políticas alimentarias para madres beneficiarias $y$, para técnicos que diseñan e implementan dichas políticas. Se utilizó el método de la teoría fundamentada. Las técnicas de recolección fueron la entrevista semi-estructurada y el grupo focal. Los participantes fueron beneficiarias de algún programa de alimentación y nutrición, con una experiencia minima de dos años $y$; los técnicos con cinco años o más de desempeño en el diseño y/o implementación de las politicas. Las políticas alimentarias se conciben y se gestionan bajo una lógica de mercado, la cual requiere de una institucionalidad que le sea funcional al modelo mercantil para proveer servicios públicos, lo cual se materializa en el esquema de la subcontratación a terceros, especialmente empresas privadas, quienes implementan estas políticas con el propósito de lograr la rentabilidad financiera. El mercado se impone como el referencial de las acciones del Estado, por tanto, se configura un Estado que limita su función a la fiscalización de las acciones subcontratadas, y las politicas de alimentación se constituyen en dispositivos para la desviación de recursos públicos hacia el sector privado.

Palabras clave Estado, Programas y politicas de nutrición y alimentación, Nutrición en salud pública, Asistencia Alimentaria; tercerización 


\section{Introducción}

Según la Organización para la Alimentación y la Agricultura de las Naciones Unidas (FAO), 795 millones de personas en el mundo, de las cuales 34,3 millones de América Latina y el Caribe, padecieron de hambre crónica en el período 2014$2016^{1}$. Durante los 20 años trascurridos desde el período base de 1990-1992, la reducción del hambre ha sido de sólo $21,4 \%{ }^{1}$. Por su parte, Colombia se enfrenta a un gran desafío, porque sigue sin resolver los fenómenos de los déficits de una proporción importante de sus niños y jóvenes, expresado principalmente, en retraso en el crecimiento $y$, en el déficit de micronutrientes como el hierro, el zinc y la vitamina $\mathrm{A}^{2}$; al tiempo que el País se ve abocado al fenómeno de las Enfermedades Crónicas No Transmisibles (ECNT), las cuales afectan en gran medida a la población adulta $^{3}$. Esta doble carga de la malnutrición ${ }^{4}$ se presenta en medio de una situación de pobreza y de profunda desigualdad en la distribución de la riqueza, lo cual genera un gradiente social que se expresa en las inequidades en el estado nutricional, dado que la prevalencia del retraso en el crecimiento en los menores de cinco años del nivel 1 del Sisbén (Sistema de Identificación de Potenciales Beneficiarios de Programas Sociales) fue de $16,8 \%$; mientras que en el nivel 4 o más fue de $9,1 \%^{2}$.

Frente a este panorama, las Políticas y Programas de Alimentación y Nutrición -PPAN- constituyen la respuesta del Estado para afrontar la dualidad nutricional que padece la población. No obstante, en el marco de un modelo de desarrollo neoliberal, de globalización y, de mayor posicionamiento de los intereses del sector privado y de transnacionales, estas políticas pueden actuar como mecanismos que legitiman una concepción residual del Estado y por tanto, de lo social $^{5,6}$.

Aunque el hambre y la inseguridad alimentaria han ganado un lugar privilegiado en la agenda pública y como resultado han proliferado una diversidad de políticas para enfrentar dichos fenómenos, la situación alimentaria y nutricional de la población no mejora en la misma medida. Asimismo, los estudios que evalúan los efectos de las PPAN son predominantemente de corte cuantitativo $^{7-10}$, en términos de variables relacionadas con el consumo de alimentos, antropométricas y bioquímicas; pero poco se ha indagado sobre la experiencia de los sujetos que interactúan con las PPAN. Es por ello que esta investigación tuvo como objetivo comprender los significados de las políticas de alimentación y nutrición para un grupo de beneficiarias, así como para los técnicos, involucrados en su diseño e implementación.

Los hallazgos presentados en este manuscrito hacen parte de una investigación marco titulada "Los significados en tensión de las políticas de alimentación y nutrición para un grupo de beneficiarias técnicos en dos Municipios de Antioquia"11.

\section{Metodología}

Se utilizó el método de la teoría fundamentada -TF-, basado en el interaccionismo simbólico ${ }^{12}$. Asimismo, se acogió el enfoque constructivista de la TF de Charmaz, el cual asume una epistemología relativista, mira el conocimiento como socialmente producido, reconoce múltiples visiones tanto de los participantes como del investigador y adopta una posición reflexiva hacia las acciones y situaciones propias y de los participantes, así como de las construcciones analíticas que hacemos los investigadores. La perspectiva constructivista considera la investigación como un proceso y un producto, el cual se desarrolla bajo condiciones preexistentes, surge de situaciones emergentes, y está influenciado por las perspectivas, privilegios, ubicación, interacción y ubicación geográfica de los investigadores ${ }^{13}$.

Charmaz define la TF como un método de investigación cualitativa en el cual la recolección y el análisis se informan recíprocamente y se moldean el uno al otro mediante un proceso iterativo emergente. El método comparativo y el proceso iterativo permiten que el investigador interactúe con los datos y con los análisis emergentes al formularse preguntas analíticas durante todo el proceso, lo cual lleva a que los investigadores eleven sus análisis hacia niveles más abstractos e intensifiquen su poder ${ }^{14}$.

Las técnicas que se emplearon en la presente investigación fueron la entrevista semi-estructurada y el grupo focal. Desde una perspectiva constructivista Guber considera que, la entrevista "es una relación social de manera que los datos que provee el entrevistado son la realidad que éste construye con el entrevistado en el encuentro $^{15 "}$.

Por su parte, la técnica grupal permite la expresión de las creencias y actitudes que subyacen o están en la base del comportamiento. Los datos con respecto a las percepciones y opiniones son enriquecidos/cualificados a través de la interacción del grupo, dado que la participación indivi- 
dual puede ser realzada o mejorada en un grupo determinado $\mathrm{o}^{16,17}$.

$\mathrm{El}$ análisis fue orientado mediante el proceso de codificación y de categorización, consistente en tres momentos iterativos: codificación abierta, codificación axial y codificación selectiva ${ }^{13,18}$. El estudio fue aprobado por el Comité de Ética de la Facultad Nacional de Salud Pública de la Universidad de Antioquia.

Los temas que orientaron las entrevistas fueron: actividades cotidianas de los participantes, cómo sienten y viven las personas el programa al que pertenecen $y$, las oportunidades que brinda el programa a los miembros del hogar. A medida que se realizó el análisis y el muestreo teórico, se afinaron las categorías que orientaron la recolección de la información, como producto de este proceso la guía de entrevista se ajustó progresivamente.

Los participantes fueron: a) madres beneficiarias de las PPAN de la ciudad de Medellín y del Municipio de Angostura, Antioquia - Colombia, cuyos hijos hubieran pertenecido, por al menos dos años, a alguna de las PPAN de su localidad y, b) profesionales que hubieran tenido como mínimo cinco años de experiencia desempeñándose como técnicos operadores, coordinadores o diseñadores de alguna PPAN, del ámbito local, departamental y nacional. En el Cuadro 1 se hace una descripción de las PPAN a las que pertenecían los participantes del estudio. Los primeros contactos con las beneficiarias y con los técnicos entrevistados, se hicieron gracias a que los investigadores los conocían previamente, los demás participantes fueron seleccionados mediante la técnica de bola de nieve. A todos los participantes se les informó en detalle los objetivos y características del estudio y, se les solicitó el Consentimiento Informado. Todas las entrevistas fueron grabadas y luego trascritas en verbatim. En el Cuadro 2 y la Tabla 1 se presentan las características sociodemográficas de los participantes, según el tipo de entrevista (I: individual; G: entrevista grupal, inicialmente planeada como individual pero que en la práctica se desarrolló con varios participantes y; GF: grupo focal).

El trabajo de recolección, de análisis y de interpretación de la información, se realizó en dos fases. La primera fase consistió en la realización de 12 entrevistas; 10 estuvieron dirigidas a madres beneficiarias $y$, las restantes a coordinadores de las PPAN. A medida que se avanzó en la recolección de la información, se inició el microanálisis de cada entrevista trascrita y se realizó la codificación abierta, producto de lo cual se ob- tuvieron 1.276 códigos, los cuales se organizaron en un archivo de Excel y se clasificaron por temas. Durante el proceso también, se construyeron 54 memos, los cuales constituyeron pistas importantes para la segunda fase de trabajo de campo, así como para la formulación de algunos supuestos preliminares. Se construyeron categorías a partir de la agrupación de códigos que daban cuenta de sucesos, acontecimientos y acciones, similares en su naturaleza o, que estuvieran relacionados por el significado, de modo que las categorías correspondieron a ideas analíticas que representaban los fenómenos que emergían de los datos ${ }^{13,18}$.

Producto de este momento de análisis, emergieron unas categorías iniciales y para develar las relaciones entre la estructura y los procesos $\mathrm{o}$, dicho de otra manera, para explicar las acciones e interacciones que protagonizan los sujetos en su diario vivir con las políticas de alimentación y nutrición, se construyó un esquema llamado "matriz paradigmática", una herramienta que propone la TF. Un ejemplo de una matriz paradigmática basada en un fenómeno emergente producto del proceso de análisis se presenta en la Figura 1. El segundo momento del análisis, consistió en el refinamiento de las matrices paradigmáticas mediante un proceso de lectura y relectura analítica de los datos. El proceso de análisis alcanzado hasta el momento orientó, mediante muestreo teórico, la realización de 13 entrevistas más y un grupo focal. Siete de las entrevistas, fueron dirigidas a técnicos que operaban las políticas y programas y, seis a madres beneficiarias de tres programas de alimentación y nutrición. Del grupo focal participaron 13 madres, este encuentro tuvo como propósito hacer devolución de los hallazgos y además, escuchar aportes de las participantes. Como producto del microanálisis de esta fase, se obtuvieron 3.415 códigos, los cuales fueron allegados a la codificación axial inicialmente hecha, a partir del método de la comparación constante se avanzó hacia la codificación selectiva de la cual se obtuvo la categoría principal, así como las subcategorías interrelacionadas, que se presentan en este manuscrito (Figura 2).

\section{Resultados}

El análisis de los datos permitió comprender que las PPAN se conciben y se gestionan bajo una lógica de mercado, este fenómeno se soporta en tres subcategorías interrelacionadas: a) institucionalidad funcional al modelo mercantil; b) tercerización de todas las fases de las PPAN y; c) 
Cuadro 1. Políticas y programas de alimentación y nutrición a los que pertenecían los participantes del estudio.

\begin{tabular}{|c|c|c|c|}
\hline Ámbito & $\begin{array}{l}\text { Políticas y Programas de } \\
\text { alimentación y nutrición }\end{array}$ & Población objeto & Tipo de intervención \\
\hline \multirow[t]{4}{*}{ Nacional } & \multirow[t]{3}{*}{$\begin{array}{l}\text { Política de Estado para el } \\
\text { Desarrollo Integral de la } \\
\text { Primera Infancia: de Cero a } \\
\text { Siempre (Ley } 1804 \text { de 2016). }\end{array}$} & $\begin{array}{l}\text {. Mujeres gestantes. } \\
\text {. Mujeres lactantes. } \\
\text {. Menores de } 2 \text { años. }\end{array}$ & $\begin{array}{l}\text { Modalidad Entorno Familiar: } \\
\text { procesos pedagógicos de formación } \\
\text { y acompañamiento para la familia, } \\
\text { cuidadores y mujeres gestantes. Pertenece } \\
\text { a esta modalidad el tradicional programa } \\
\text { de Familia, Mujer e Infancia -FAMI-. }\end{array}$ \\
\hline & & $\begin{array}{l}2 \text { años hasta } 5 \text { años de } \\
\text { edad. }\end{array}$ & $\begin{array}{l}\text { Modalidad Entorno Institucional. Los } \\
\text { niños son asistidos en unas guarderías } \\
\text { llamadas Centros de Desarrollo Integral } \\
\text {-CDI-. Reciben atención pedagógica por } \\
\text { parte de profesionales y alimentación. Las } \\
\text { jornada puede ser de } 5 \text { u } 8 \text { horas diarias. }\end{array}$ \\
\hline & & $\begin{array}{l}2 \text { años hasta } 5 \text { años de } \\
\text { edad. }\end{array}$ & $\begin{array}{l}\text { Modalidad Comunitaria: Hogares } \\
\text { Comunitarios de Bienestar Familiar } \\
\text {-HCBF-. Los niños asisten por ocho } \\
\text { horas al día a una casa de una madre de } \\
\text { la comunidad, conocida como madre } \\
\text { comunitaria, allí realizan algunas } \\
\text { actividades lúdicas y reciben alimentación }\end{array}$ \\
\hline & $\begin{array}{l}\text { Familias en Acción (Red de } \\
\text { Protección Social contra la } \\
\text { Extrema Pobreza. Documento } \\
\text { del Consejo Nacional de Política } \\
\text { Social \# } 102 \text { de 2.006). }\end{array}$ & Menores de 6 años. & $\begin{array}{l}\text { Transferencia económica a la madre } \\
\text { del niño beneficiario, condicionada a la } \\
\text { asistencia al programa de crecimiento } \\
\text { y desarrollo en una institución de } \\
\text { atención en salud. }\end{array}$ \\
\hline \multirow[t]{2}{*}{$\begin{array}{l}\text { Provincial } \\
\text { y local }\end{array}$} & $\begin{array}{l}\text { Plan de Mejoramiento } \\
\text { Alimentario y Nutricional de } \\
\text { Antioquia -MANA- (Ordenanza } \\
\text { \# } 46 \text { de } 2.016 \text { de la Asamblea } \\
\text { Departamental de Antioquia). }\end{array}$ & Menores de 6 años. & $\begin{array}{l}\text { Entrega un subsidio alimentario } \\
\text { consistente en galletas y leche } \\
\text { fortificadas. También realiza valoración } \\
\text { antropométrica. }\end{array}$ \\
\hline & $\begin{array}{l}\text { Buen Comienzo Antioquia } \\
\text { (Ordenanza \# } 26 \text { de } 2.015 \text { de } \\
\text { la Asamblea Departamental de } \\
\text { Antioquia) y Buen Comienzo } \\
\text { Medellín (Acuerdo \# } 14 \text { de } \\
\text { 2.004 del Concejo de Medellín). }\end{array}$ & $\begin{array}{l}\text {. Gestación hasta } 23 \\
\text { meses de edad. } \\
\text {. Gestación hasta cinco } \\
\text { años en zona rural. } \\
\text {. Gestación hasta } \\
\text { tres años en } \\
\text { complejo carcelario y } \\
\text { penitenciario. }\end{array}$ & $\begin{array}{l}\text { Modalidad Entorno Familiar: promueve } \\
\text { el desarrollo integral potenciando el rol } \\
\text { educativa de la familia y los cuidadores. } \\
\text { Realiza encuentros educativos grupales } \\
\text { de nutrición, asesorías familiares, } \\
\text { valoración antropométrica y entrega de } \\
\text { paquetes alimentarios. }\end{array}$ \\
\hline
\end{tabular}

Fuente: elaboración propia.

operatividad centrada en la rentabilidad financiera (Figura 2).

\section{Institucionalidad funcional al modelo mercantil}

La institucionalidad se moldeó al esquema de mercado mediante un entramado de condiciones políticas, económicas y administrativas, que le imponían una serie de restricciones para implementar de forma directa las PPAN. La pri- mera se materializa en la legislación colombiana relacionada con la contratación pública que data de 1.993, la cual abocaba a las instituciones a recurrir a la figura de intermediarios, mediante la subcontratación de instituciones principalmente privadas como operadores de la política o programa.

Otro elemento consistía en que las principales PPAN del país, estaban tuteladas por algunos organismos internacionales -OI-. Este aspecto tenía un trasfondo económico debido a que una 
Cuadro 2. Características sociodemográficas de las madres entrevistadas, beneficiarias de los programas de alimentación y nutrición de Medellín y Angostura, Antioquia.

\begin{tabular}{|c|c|c|c|c|c|c|c|}
\hline \multicolumn{8}{|c|}{ Características } \\
\hline $\begin{array}{c}\# \\
\text { entrevista }\end{array}$ & $\begin{array}{l}\text { Tipo de } \\
\text { entrevista }\end{array}$ & $\begin{array}{l}\text { Zona de } \\
\text { residencia }\end{array}$ & Edad & $\begin{array}{l}\text { Nivel } \\
\text { Sisbén }\end{array}$ & $\begin{array}{c}\text { Nivel } \\
\text { educativo }\end{array}$ & $\begin{array}{l}\text { Rol en el } \\
\text { programa }\end{array}$ & $\begin{array}{c}\text { Participa } \\
\text { en varios } \\
\text { programas }\end{array}$ \\
\hline 1 & I & Urbana & 20 & 1 & Secundaria & Beneficiaria & No \\
\hline 4 & I & Urbana & 41 & 2 & Secundaria & Madre líder & No \\
\hline 5 & I & Rural & 43 & 2 & Primaria & Madre líder & $\mathrm{Si}$ \\
\hline 7 & I & Rural & 58 & 1 & Primaria & Beneficiaria & $\mathrm{Si}$ \\
\hline 8 & I & Rural & 41 & 1 & Primaria & Beneficiaria & $\mathrm{Si}$ \\
\hline 9 & I & Rural & 41 & 2 & Primaria & Beneficiaria & $\mathrm{Si}$ \\
\hline 10 & I & Rural & 35 & 1 & Primaria & Beneficiaria & $\mathrm{Si}$ \\
\hline 11 & I & Urbana & 18 & 1 & Técnica & Beneficiaria & $\mathrm{Si}$ \\
\hline 12 & I & Urbana & 35 & 2 & Técnica & Beneficiaria & $\mathrm{Si}$ \\
\hline 14 & I & Urbana & 18 & 1 & Técnica & Beneficiaria & No \\
\hline 15 & I & Urbana & 48 & 1 & Primaria & Beneficiaria & No \\
\hline 16 & I & Urbana & 38 & 2 & Secundaria & Beneficiaria & $\mathrm{Si}$ \\
\hline 17 & I & Urbana & 42 & 2 & Técnica & Beneficiaria & $\mathrm{Si}$ \\
\hline 18 & I & Urbana & 53 & 1 & Primaria & Beneficiaria & No \\
\hline 19 & I & Urbana & 32 & 1 & Técnica & Beneficiaria & No \\
\hline 20 & I & Rural & 23 & 1 & Técnica & Beneficiaria & $\mathrm{Si}$ \\
\hline \multirow[t]{4}{*}{2} & G & Urbana & 35 & 1 & Primaria & Beneficiaria & $\mathrm{Si}$ \\
\hline & G & Urbana & 54 & 1 & Ninguno & Beneficiaria & $\mathrm{Si}$ \\
\hline & G & Urbana & 77 & 2 & Primaria & Beneficiaria & $\mathrm{Si}$ \\
\hline & G & Urbana & 35 & 1 & Ninguno & Beneficiaria & $\mathrm{Si}$ \\
\hline \multirow[t]{14}{*}{26} & GF & Rural & 28 & 1 & Secundaria & Beneficiaria & No \\
\hline & GF & Urbana & 32 & 1 & Técnica & Beneficiaria & No \\
\hline & GF & Urbana & 20 & 1 & Técnica & Beneficiaria & No \\
\hline & GF & Urbana & 21 & 1 & Técnica & Beneficiaria & No \\
\hline & GF & Urbana & 26 & 1 & Técnica & Beneficiaria & No \\
\hline & GF & Rural & 31 & 1 & Secundaria & Beneficiaria & No \\
\hline & GF & Rural & 21 & 1 & Técnica & Beneficiaria & No \\
\hline & GF & Rural & 43 & 1 & Primaria & Beneficiaria & No \\
\hline & GF & Rural & 23 & 1 & Primaria & Beneficiaria & No \\
\hline & GF & Rural & 35 & 1 & Primaria & Beneficiaria & No \\
\hline & GF & Urbana & 31 & 1 & Primaria & Beneficiaria & No \\
\hline & GF & Rural & 32 & 2 & Primaria & Beneficiaria & No \\
\hline & GF & Rural & 22 & 1 & Secundaria & Beneficiaria & No \\
\hline & GF & Urbana & 19 & 1 & Secundaria & Beneficiaria & No \\
\hline
\end{tabular}

I: entrevista individual; G: entrevista grupal; GF: grupo focal.

de las políticas de mayor cobertura en el país, era financiada mediante los créditos provenientes de la banca internacional.

Otro ejemplo, lo constituyen los tratados de libre comercio. Los relatos de algunos técnicos validaban la idea de un individuo racional, el cual decide maximizando sus intereses al menor precio, desde esta perspectiva, la política era vista como un mecanismo para incentivar la libertad del consumidor. No se visualizaba una preocupación por la dependencia de las importaciones de alimentos, sino que prevalecía la preocupación de no incumplir los acuerdos comerciales:

[...] cerrar las importaciones, sería como no tener flujo adecuado de alimentos, y sé que el Gobierno, es decir, el Presidente y demás dirigen- 
Tabla 1. Características sociodemográficas de los técnicos entrevistados, vinculados a las políticas y programas de alimentación y nutrición, en los niveles municipal, departamental y nacional.

\begin{tabular}{|c|c|c|c|c|c|c|}
\hline $\begin{array}{c}\# \\
\text { entrevista }\end{array}$ & $\begin{array}{c}\text { Rol en la política o } \\
\text { programa }\end{array}$ & Nivel territorial & Sexo & Edad & Nivel educativo & $\begin{array}{c}\text { Años de } \\
\text { experiencia con } \\
\text { las PPAN }\end{array}$ \\
\hline 21 & Formulación & Nacional & Femenino & 54 & Universitario & $>8$ \\
\hline 22 & Formulación & Nacional & Femenino & 43 & Postgrado & $>8$ \\
\hline 23 & Formulación & Nacional & Femenino & 35 & Postgrado & $>8$ \\
\hline 24 & Formulación & Nacional & Femenino & 40 & Postgrado & $>8$ \\
\hline 3 & Coordinación & Municipal & Masculino & 41 & Universitario & $>8$ \\
\hline 6 & Coordinación & Departamental & Femenino & 38 & Postgrado & 8 \\
\hline 13 & Implementación & Municipal & Femenino & 45 & Universitario & $>8$ \\
\hline 18 & Implementación & Departamental & Femenino & 30 & Universitario & 5 \\
\hline 25 & Implementación & Municipal & Femenino & 41 & Técnica & 5 \\
\hline
\end{tabular}

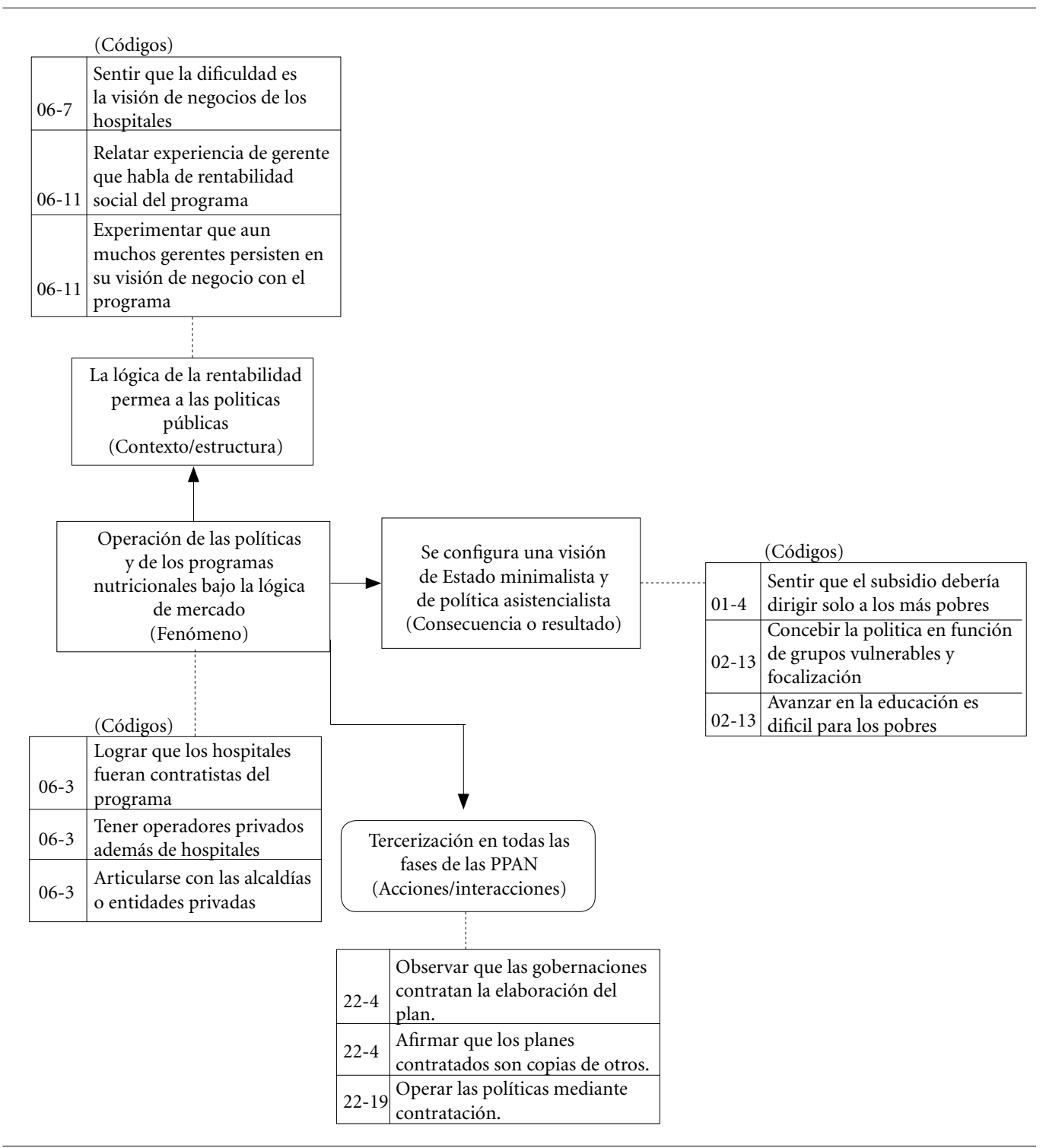

Figura 1. Ejemplo matriz paradigmática producto del processo de análisis. 


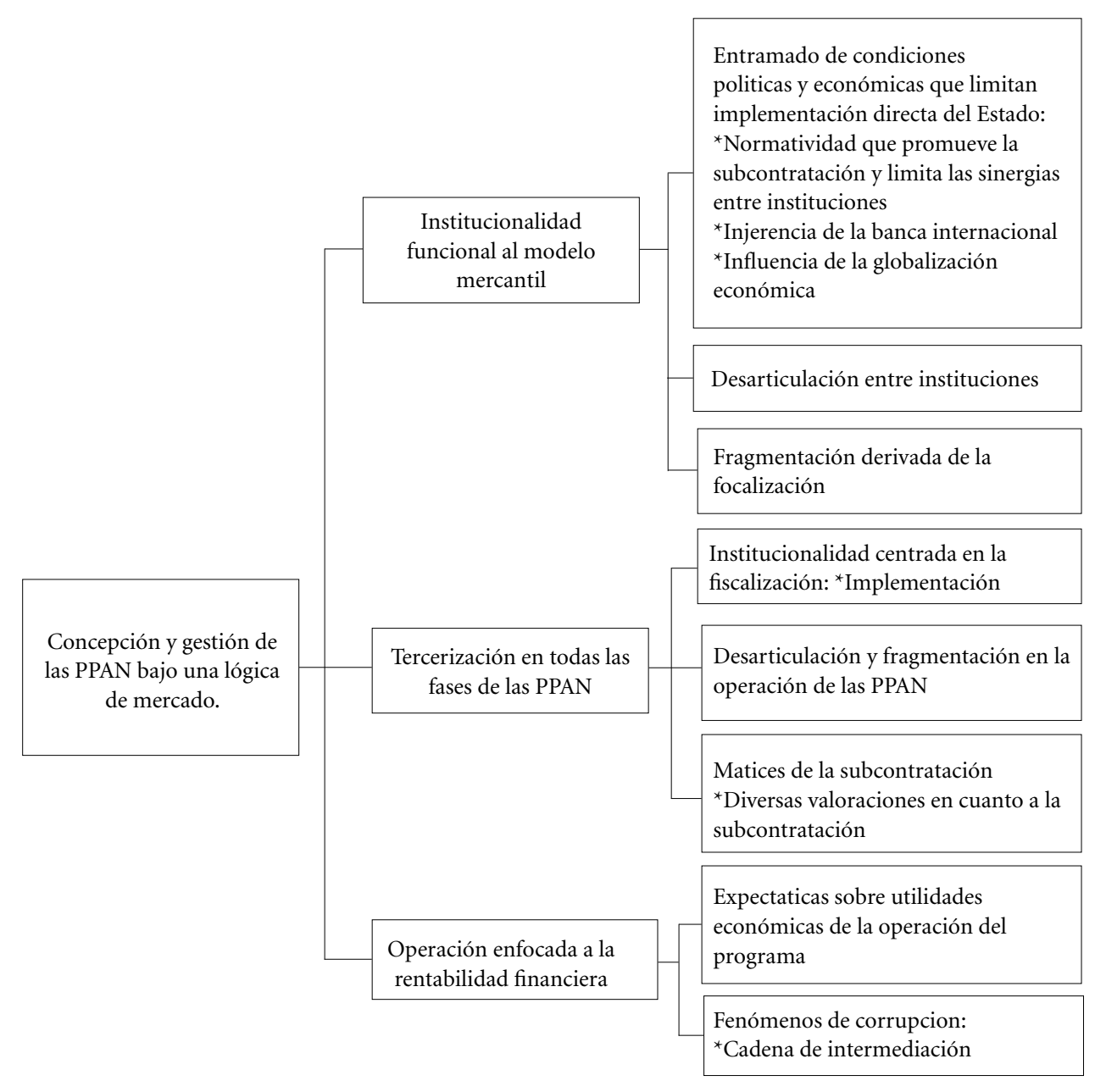

Figura 2. Categoría principal y subcategorías interrelacionadas producto de la codificacion selectiva.

tes, no están de acuerdo con estas medidas, porque ya han firmado tratados de libre comercio... (DNP-D-23,9).

La desarticulación, constituía otro elemento que contribuía a configurar una institucionalidad funcional al modelo mercantil, en la medida en que cada institución definía una población objeto de la PPAN y contrataba a terceros su ejecución, así cada sector hacia una serie de acciones puntuales de forma independiente, sin procesos para articular esfuerzos y recursos de otras instituciones y/o programas.

Otra causa para la desarticulación, la constituía los mecanismos de focalización, porque cada institución tenía un sistema propio para la identificación de su población objeto y, como producto resultaba una diversidad de sistemas de información, los cuales fragmentaban la población, de forma tal que, las políticas pocas veces confluían en un mismo territorio con una oferta integral a las comunidades.

\section{Tercerización en todas las fases de las PPAN}

En coherencia con lo expuesto antes, la forma de materializar la lógica mercantil de las PPAN era mediante la tercerización, es decir la contratación de operadores. Con este mecanismo, a muchas de las instituciones del Estado sólo les quedaba la función de hacer la interventoría de las actuaciones de los operadores. Bajo esta lógica, el rol del Estado en la implementación de la política se circunscribía a la ejecución de procesos administrativos y, a la supervisión de los 
contratistas, lo cual reñía con la posibilidad de abordar la política de forma más integral, de repensar los procesos $y$, de atender las necesidades de la población objeto. Los técnicos sentían frustración al experimentar que su ejercicio profesional se destinaba en gran medida a las tareas de la contratación y de la fiscalización, en detrimento del aporte que podrían hacer a las comunidades desde su campo de experticia. Esta preocupación fue expresada de la siguiente manera por un participante:

[...] todo eso se ha quedado de lado porque siempre lo operativo es tan complejo; revisar el uno a uno, revisar las condicionalidades, a uno se lo absorbe la operatividad, y a los otros programas sociales les pasa lo mismo, y a todos nos pasa lo mismo... (FA-D-21,18).

Otra de las implicaciones de la tercerización, consistía en que los operadores externos llegaban con su propio equipo de profesionales a las localidades, en lugar de contratar al recurso humano disponible en los territorios. También, se propiciaba que los programas privilegiaran como sus principales proveedores a la industria de alimentos, en detrimento de la provisión mediante el mercado local o mediante el incentivo a la producción de alimentos en los territorios. No obstante, la principal limitante que representaba la tercerización, era que bajo la estructura normativa del país, las acciones de política se debían ejecutar exactamente como lo establecían las condiciones del contrato. Esta situación dejaba con un estrecho margen de maniobra al mismo Estado, en el caso de que necesitara reorientar los recursos para asistir a la población ante situaciones especiales o de crisis. Asimismo, estos mecanismos de contratación tenían poca posibilidad de adaptarse a las particularidades de comunidades que tenían dinámicas particulares, como las que dependen de la minería o las minorías étnicas. De esta forma analizaba un técnico las implicaciones de la modalidad más utilizada de contratación:

[...] la licitación pública es la que se ha considerado más transparente para nosotros, con el agravante de que la licitación tú no la puedes cambiar, jes o es!, no permite adaptarse a lo que encuentres en el territorio... En una comunidad indígena, donde no hay agua y tú dijiste que ibas hacer una huerta, al operador le toca hacer una huerta, una huerta que va durar un mes porque no hay agua, entonces la licitación tiene esa restricción (DPS-D-22,19).

Es importante anotar que tanto los técnicos como las beneficiarias, tuvieron algunas valora- ciones positivas frente a la contratación de terceros. Los técnicos expresaban que la contratación de operadores posibilitaba mayor cobertura, dado que el Estado tenía una capacidad de respuesta limitada. Por su parte, las madres destacaban como positivo que recibían intervención de profesionales de diferentes áreas; asimismo, consideraban que las características nutricionales, organolépticas y de inocuidad de los alimentos eran mejores. Una beneficiaria manifestaba así su experiencia:

[...] entonces es muy agradable porque aprendo demasiado, entonces es bueno, porque uno mismo se va corrigiendo, es agradable, porque, le ayuda a uno para bien. Creo que sin el Programa la vida de uno sería un poquito más desastrosa, claro, porque, le enseñan a uno: "se debe hacer esto, intente a hacer esto, procure hacer esto" (MA-M-11,13).

\section{Operatividad centrada en la rentabilidad financiera}

La figura de la tercerización tenía como efecto que la implementación de las PPAN se centrara en el interés por la rentabilidad económica. Un operador lo expresaba de la siguiente forma:

[...] Es que a mí solo me queda el dos por ciento de ganancia y eso [el programa] me tendría que dejar el diez por ciento (BC-PC-06,11).

El tema más complejo derivado del interés económico, reside en el fenómeno de corrupción. La figura más común a la que acudían algunos operadores consistía en conformar organizaciones que se especializaban en obtener los contratos ofrecidos mediante las licitaciones públicas, muchas de las cuales, no cumplían con las especificaciones del contrato y por tanto, cometían graves irregularidades en el suministro del subsidio de alimentos. Los técnicos mencionaban que estas "entidades fachadas" eran expertas en eludir las sanciones judiciales y tenían la pericia de aplicar continuamente a las licitaciones, porque recurrían a ciertas estrategias como asociarse con otros y cambiar frecuentemente de razón social. Un técnico relataba algunas de los mecanismos que adoptaban los operadores de la siguiente forma:

[...] Los operadores lo que hacen en muchos casos es que tienen una razón social en un lugar determinado, pero arman una unión temporal, entonces toman otro nombre. $Y$ si tienen impedimentos, se unen con otra persona y se cambian el nombre, así ya no tienen problema en presentarse a las licitaciones públicas porque aparecen con otra razón social (DPS-D-22,25). 


\section{Discusión}

La creciente imposición del mercado como el referente del Estado. En el proceso de transformación del Estado de Bienestar en el Estado transnacional, se sentaron las bases para la instauración de la clase capitalista transnacional, con la cual devino una nueva política social y las medidas de globalización económica. Este viraje neoliberal, ocasionó que la globalización fuera el referencial, es decir, la nueva norma para la configuración y gestión de las políticas públicas, con una importancia creciente del "mercado", 19 . Hasta tal punto fue la penetración de la corriente neoliberal, que las decisiones de los Estados y de las transnacionales son en la práctica, casi idénticas ${ }^{20}$. No obstante, el Estado transnacional requiere la continuidad del Estado-nación, porque en éste tienen lugar y se legitiman las decisiones que materializan la globalización ${ }^{21}$. El contexto de mercado y, por tanto, neoliberal, configuró dos fenómenos que se pretenden discutir a continuación: configurar un Estado circunscrito a la fiscalización de acciones y, constituir las políticas públicas de alimentación y nutrición en dispositivos para la desviación de recursos públicos al sector privado.

Configuración de un Estado circunscrito a la fiscalización de acciones. Con las reformas del Estado y la subsecuente posicionamiento de un modelo de mercado que amplía la participación de sector privado como operador en la provisión de servicios sociales, la principal actuación de las instituciones estatales se circunscribe a la fiscalización de los operadores, por tanto, emerge un Estado check-list, el cual dispone de todo su acervo institucional para la constatación del cumplimiento de las actividades que ejecutan terceros contratados. Este tipo de Estado es el resultado del modelo económico neoliberal, el cual promulga una participación restringida de éste en diversas dimensiones, en especial en las políticas públicas sociales, fenómeno que han experimentado otros países de América Latina como Brasil22,23.

Estado check-list, contrario a la imagen que nos ha vendido el modelo neoliberal de un Estado mínimo o adelgazado, se soporta en un gran andamiaje burocrático que paulatinamente ha migrado su actuación de la implementación directa de la política hacia la fiscalización, esta última no debe homologarse a menor necesidad de recursos humanos, logísticos y económicos, sino que por el contrario, se ha visto en la necesidad de implementar estrategias cada vez más refinadas para cumplir su rol fiscalizador, en la medida en que la subcontratación se ha convertido en un fortín económico para los operadores y, por tanto, esto ha exacerbado la preocupación por los inadecuados manejos de los recursos públicos, situación que se puede leer entre líneas de los relatos de los beneficiarios y de los técnicos entrevistados para esta investigación.

La institucionalidad acude al artilugio de fragmentar y de compartimentar la problemática social, en consecuencia, se especializa en convertir fenómenos complejos en un paquete de acciones, de esta forma traduce la problemática alimentaria al lenguaje de las licitaciones, la cual constituye el mecanismo de transacción entre el Estado y los terceros. Esto explica en gran medida la desarticulación entre las instituciones, la cual era percibida por los participantes de este estudio, como uno de los principales escollos de las PPAN, de la cual se derivaban situaciones como la duplicidad de acciones y la sobre-intervención a los beneficiarios. La desarticulación era vista como la raíz de la ineficiencia de las políticas, no obstante, este estudio permite comprender que constituye una condición inherente para el despliegue del modelo mercantil. Este fenómeno también es reportado por algunos autores en México y Chile. En el primer caso, se plantea que la desarticulación de la política pública de alimentación produce duplicidad de programas, estrategias mal concebidas y desperdicio de recurso ${ }^{24}$. Por su parte Salinas y Vio, advierten sobre la desarticulación existente entre los ministerios de salud y de educación de Chile para la ejecución de la Política de Promoción de Salud Escolar, pese a los diversos acuerdos políticos entre ambos sectores para llevar a cabo un trabajo coordinado. Los autores chilenos concluyen que existe una brecha entre el diagnóstico sobre la situación de salud de la población infantil y juvenil y la respuesta de la oferta programática para darle respuesta efectiva a dicha problemática ${ }^{25}$.

En medio de este panorama emerge la cuestión de cómo a medida que las instituciones estatales se vuelcan hacia la fiscalización, pierde terreno el papel del Estado de repensar políticas integrales y universales, con las cuales se supere la tendencia asistencialista que se traduce en la entrega de un subsidio para suplir unas necesidades mínimas de calorías y de nutrientes. Asimismo, surge la preocupación por la pérdida de un Estado que se centra en la fiscalización, en lugar de la garantía de los derechos sociales. Esa mutación que sufren las funciones del Estado, permite colegir que las instituciones gubernamentales están más interesadas en su propia permanencia que en 
proveer la atención de su población objeto. Por tanto, la configuración vertical y desarticulada de las instituciones se expresa en la incapacidad e indiferencia de éstas para abordar de forma integral los fenómenos sociales que afectan a las familias.

Desviación de recursos públicos al sector privado mediante el dispositivo de PPAN. El primer elemento para explicar el mecanismo de desviación de los recursos públicos hacia el sector privado mediante las políticas públicas de alimentación y nutrición, radica en el reordenamiento de los límites entre de lo público y lo privado. En la medida en que el nuevo referencial impone un interés en fortalecer la empresa privada ${ }^{5,6}$, el Estado entrega la implementación de un segmento importante de sus responsabilidades sociales al sector privado.

El segundo elemento, es la injerencia de los Organismos Internacionales -OI- en las decisiones del sector público. Los OI han tenido un papel decisivo en el diseño de políticas públicas porque muchos de ellos han hecho de la lucha contra la pobreza una de sus principales finalidades y por consiguiente, como plantea Pérez, han movilizado importantes recursos para la realización de investigaciones que ofrezcan conocimientos y estadísticas alrededor de la dialéctica desarrollo/ subdesarrollo ${ }^{26}$. Entidades como el Banco Mundial -BM-, la Organización Mundial del Comercio y el Fondo Monetario Internacional, tuvieron un papel determinante en la nueva configuración de las políticas públicas de salud ${ }^{20}$ y por tanto, en las de alimentación y nutrición no solo en América Latina sino en la mayoría de los países denominados como de bajos ingresos, como fue el caso de Bangladesh donde el BM se convierte en el administrador de los fundos donados por diferentes entidades internacionales para la operación del Plan del Servicio Nacional de Nutrición cuyo principal objetivo es el mejoramiento de la alimentación de la primera infancia ${ }^{27}$. El BM impulsó la receta de la reestructuración del sector salud en los denominados países en desarrollo, con una fuerte impronta en el fortalecimiento del sector privado, aspecto que quedó plasmado en el emblemático informe "Invertir en salud" de $1.993^{28}$. Otra estrategia utilizada por los OI para la desviación de los recursos públicos a privados, ha sido mediante mecanismos como los tratados de libre comercio, los cuales han jugado un papel determinante en la configuración de las políticas de alimentación y nutrición en el país. Para ilustrar este hecho está el caso de la creciente importación de maíz desde Los Estados Unidos por parte de Colombia $^{29}$, pese a que este alimento representa uno de los productos autóctonos y constitutivos de la cultura alimentaria y, que el país posee las condiciones para lograr la producción para abastecer a toda la población. La injerencia de los OI, también han promovido algunas modalidades soterradas de desviación de los recursos públicos al sector privado, como ha sido el impulso de los programas de transferencias condicionadas en efectivo -PTCE- en América Latina y, mediante el papel protagónico de las multinacionales de alimentos. No es coincidencia que la expansión de los PTCE se haya iniciado en la década de los noventa, período en el que tuvieron su mayor despliegue las medidas neoliberales, en 1.997 solo tres países implementaban PTCE; en 2010 tenían presencia en 18 países $^{30}$. En la mayoría de los países los PTCE dependen de los OI, como es el caso de Colombia donde el 54\% del subsidio económico entregado a las familias mediante el Programa de Familias en Acción, provenía de créditos realizados al BM y al Banco Interamericano de Desarrollo ${ }^{31}$. Las multinacionales de alimentos también se convierten en destinatarias de los recursos públicos, en la medida en que un número importante de las PPAN incorporaban alimentos procesados producidos por multinacionales, los cuales se entregan a los beneficiarios como parte de los subsidios.

El tercer elemento para destacar, es la proliferación de intermediarios, principalmente privados para la ejecución de las acciones de política pública. Los preceptos implantados por el modelo neoliberal, consistían en que para aumentar la eficiencia: a) se debía separar la financiación de la prestación del servicio, b) que era necesario promover la competencia entre las organizaciones, las instituciones y los sectores públicos y privados, c) que la administración privada era más eficiente y menos corrupta que la pública y d) que se debía propender por el subsidio a la demanda y no a la oferta ${ }^{20}$. Desde esta perspectiva, los operadores se convertirían en una extensión del Estado, e implementarían diversidad de acciones y estrategias tales como las PPAN; igualmente proliferaría un amplio menú de entidades con injerencia en todo el espectro de las políticas, desde su diseño, abarcando su implementación y su evaluación. Varios autores analizan cómo entidades no gubernamentales tienen un papel activo en la ejecución de las políticas sociales, en particular las de salud y nutrición no sólo en América Latina sino en otros continentes como el africano y el asiático ${ }^{32-36}$.

Durante el período de desarrollo de la presente investigación hubo denuncias frente a las graves 
irregularidades en la atención de la población infantil por parte de operadores privados, las cuales evidenciaban que dichas entidades habían encontrado en la operación de los programas, una fuente de enriquecimiento, al tiempo que no cumplían con la función para la cual fueron contratados. Lo más preocupante es que se configuran redes de corrupción política que logran legitimación social debido a que en la búsqueda de su permanencia, estas estructuras acuden a estrategias como cumplir con ciertos objetivos de alto desempeño gubernamental, de modo que se forja una suerte de "corrupción benevolente", lo cual asociado a las condiciones sociales del país configuran un escenario de trampas de pobreza dado que estas redes de corrupción tienen mayor capacidad de agencia en los territorios marginales ${ }^{37}$. Algunos reportes demuestran que durante el período comprendido entre los años 2.009 a 2.016 se registraron 3.966 casos en el sistema penal oral acusatorio relacionados con diversas formas de corrupción ${ }^{37}$. Asimismo, un informe oficial develó que en el país existían 10 redes, denominadas como "mallas empresariales de alimentación”, conformadas por consorcios y uniones temporales, integradas por entidades sin ánimo de lucro y empresas con ánimo de lucro, las cuales movían la no despreciable suma de USD \$132 millones (según tasa de cambio de noviembre de 2016) por concepto de la operación de programas de alimentación ${ }^{38}$.

\section{Conclusiones}

El mercado se impone como el referente de las acciones del Estado y por tanto, éste pierde terreno como garante de los derechos sociales para convertirse en un "Estado check-list". La institucionalidad materializa la lógica mercantil mediante procesos de tercerización, los cuales abarcan todas las fases de las políticas de alimentación y nutrición, finalmente, las políticas se configuran en dispositivos para la desviación de recursos públicos al sector privado.

\section{Colaboradores}

LPM López, CEY Delgado y GM Marín han participado en todo el proceso de construcción del manuscrito, lo cual implica contribuciones en el diseño del trabajo, análisis de la información, redacción y aprobación de la versión final y, por tanto, los autores aprobamos su contenido.

\section{Agradecimientos}

Los autores agradecemos a la Facultad Nacional de Salud Pública de la Universidad de Antioquia y al Grupo de Investigación de Gestión y Políticas en Salud por el apoyo y el financiamiento suministrado para el desarrollo de la investigación en mención.

\section{Referencias}

1. Food and Agriculture Organization (FAO), Fondo Internacional de Desarrollo Agricola (FIDA), Programa Mundial de Alimientos (PMA). El estado de la inseguridad alimentaria en el mundo 2015 [Internet]. Roma, Italia; 2015. [citado 2016 Oct 23]. Disponible en: http://www.fao.org/3/a-i4646s.pdf

2. Instituto Nacional de Bienestar Familiar (ICBF), Min. Protección Social, Instituto Nacional de Salud (INS). Encuesta Nacional de la Situación Nutricional 2010 [Internet]. Bogotá: ICBF, Ministerio de la Protección Social, INS; 2011. [citado 2016 Oct 23]. Disponible en: https: //www.icbf.gov.co/icbf/directorio/portel/ libreria/php/03.03081103.html

3. Organización Panamericana de Salud (OPS). Salud en la Américas 2012: volumen de países. Colombia. [Internet]. 2012. [citado 2016 Oct 23]. Disponible en: http: //www.paho.org/saludenlasamericas/index.php?option=com_docman\&task=doc_view\&gid=197\&Itemid=

4. Sarmiento O, Parra D, González S, González I, Forero A. The dual burden of malnutrition in Colombia. Am J Clin Nutr 2014; 100(6):1628S-1635S.

5. P. Tres retos para entender la acción pública hoy en día. In: Muller P. Las políticas públicas. $3^{\text {a }}$ ed. Bogotá: Universidad Externado de Colombia; 2010. p. 163-90

6. Sousa GW. Como reinventar a gestão e o funcionamento dos sistemas públicos e organizações estatais? Cien Saude Colet 2008; 13(Supl. 2):2019-2021. 
7. Fung C, McIsaac J, Kuhle S, Kirk S, Veugelers P. The impact of a population-level school food and nutrition policy on dietary intake and body weights of $\mathrm{Ca}$ nadian children. Prev Med 2013; 57(6):934-940.

8. Moffitt R, Ribar D. Rasch Analyses of Very Low Food SecurityAmong Households and Children in the Three City Study. Southern Economic Journal 2016; 82(4):1123-1146.

9. John J, Wolfenstetter S, Wenig C. An economic perspective on childhood obesity: Recent findings on cost of illness and cost effectiveness of interventions. $\mathrm{Nu}$ trition 2012; 28(9):829-839.

10. Erdöl Ş, Mazzucco W, Boccia S. Cost Effectiveness Analysis of Childhood Obesity Primary Prevention Programmes: A Systematic Review. Epidemiology Biostatistics and Public Health. 2014; 11(3):e94161-10.

11. Mancilla López L. Los signficados en tensión de las políticas de alimentación y nutrición para un grupo de beneficiarios y de técnicos en dos municipioos de Antioquia [tesis]. Medellín: Universidad de Antioquia; 2017.

12. Glaser BG, Strauss AL. Generating Theory. In: Glaser BG, Strauss AL. The Discovery of Grounded Theory: strategies for qualitative research. New York: Aldine de Gruyter; 1967. p. 21-44.

13. Charmaz K. Shifting the Grounds. Constructivist Grounded Theory Methods. In: Corbin J, Clarke A, Charmaz K, Stern P, Bowers B, Morse JM, editors. Developing Grounded Theory The Second Generation. California: Left Coast Press; 2009. p. 127-147.

14. Charmaz, K. Grounded Theory Methods in Social Justice Research. In: Denzin N, Lincoln Y, editors. The Sage Handbook of Qualitative Research. $4^{\text {th }}$ ed. Thousand Oaks: SAGE Publications Inc.; 2011. p. 359-380.

15. Guber R. La entrevista etnográfica o el arte de la "no directividad”. In: Guber R. La etnografía: método, campo y reflexividad. Bogotá: Norma; 2001. p. 75-100.

16. Krueger R. Focus Groups: A practical guide for applied research. Newbury Park: Sage; 1988.

17. Morse MJ. Critical Issues in Qualitative Research Methods. London: Sage; 1994.

18. Strauss A, Corbin J. Codificación abierta. In: Strauss A, Corbin J. Bases de la investigación cualitativa Técnicas y procedimientos para desarrollar la teoría fundamentada. Medelín: Universidad de Antioquia; 2002. p. 110-133.

19. Riojas-López C. 1989: Global History?. Iberoamericana [Internet] 2014; 14(54). [citado 2016 Oct 23]. Disponible en: http://journals.iai.spk-berlin.de/index. php/iberoamericana/article/view/134/762

20. Waitzkin H. El sentido común de la reforma a la salud. In: Waitzkin H. Medicina y salud pública al final del imperio. Bogotá: Universidad Nacional de Colombia; 2013. p. 197-208.

21. Abadía C, Oviedo D. Itinerarios burocráticos de la salud en Colombia: la burocracia neoliberal, su estado y la ciudadanía en salud. Revista Gerencia y Políticas de Salud 2010; 9(18):86-102.

22. Cardoso A. Public Policy Agenda Setting: Fiscal Austerity in Brazil. Revista de Direito Setorial e Regulatório 2018; 4(1):111-130.

23. Machado ML, Garcia CG, Soar C, Mamed GR, Machado PMO, Lacerda JT, Martins MC, Marcon MC. Adequação normativa dos planos estaduais de segurança alimentar e nutricional no Brasil. Cad Saude Publica 2018; 34(1):1-14.
24. López R, Gallardo E. Las políticas alimentarias de México: un análisis de su marco regulatorio. Estudios Socio-Jurídicos 2015; 17(1):11-39.

25. Salinas J, Vio F. Programas de salud y nutrición sin política de estado: el caso de la promoción de salud escolar en Chile. Rev chil nutr 2011; 38(2):100-116.

26. Pérez E. Los senderos de los Organismos Internacionales en la cooperación para el desarrollo: un panorama general sobre la evolución de las estrategias del Banco Mundial. Análisis Político 2016; 29(88):105125.

27. Rasheed S, Roy S, Das S, Chowdhury S, Iqbal M, Akter S, Jahan K, Uddin S, Thow AM. Policy content and stakeholder network analysis for infant and young child feeding in Bangladesh. BMC Public Health 2017; 17(2):27-38.

28. Banco Mundial (BM). Informe sobre el Desarrollo Mundial. Invertir en salud (Resumen) [Internet]. Washington: BM; 1993. [citado 2016 Oct 23]. Disponible en: http: //documents.worldbank.org/curated/ en/259121468340250256/pdf/341290spanish.pdf

29. Dirección Nacional de Estadística (DANE). Balanza comercial [Internet]. Comercio exterior. 2016. [citado 2016 Nov 26]. Disponible en: http: //www.dane.gov. co/index.php/comercio-exterior/balanza-comercial.

30. Cecchini S, Madariaga A. Programas de tranferencias condicionadas: balance de experiencias recientes en América Latina y el Caribe Santiago de Chile. Santiago: CEPAL; 2011.

31. Acción Social, DNP. El camino recorrido 10 años Familias en Accion. [Internet]. 2010. [citado 2016 Nov 26]. Disponible en: www.dnp.gov.co

32. Hofisi M. State-NGO Relations in Africa. Mediterranean Journal of Social Sciences 2013; 4(10):291-298.

33. Galway L, Corbett K, Zeng L. Where are the NGOs and why? The distribution of health and development NGOs in Bolivia. Globalization and Health 2012; $8(32): 1-13$.

34. Aldashev G, Navarra C. Development NGOS: basic facts. Annals of Public and Cooperative Economics 2018; 89(1):125-156.

35. Song Y, Fu L. Do Charitable Foundations Spend Money Where People Need It Most? A Spatial Analysis of China. Int J Geo-Inf 2018; 7(3):1-17.

36. Werker E, Ahmed F. What Do Nongovernmental Organizations Do? Journal of Economic Perspectives 2008; 22(2):73-92.

37. Gómez D. Redes de corrupción política: una revisión para el caso colombiano. Análisis Político 2018; 31(92):180-201.

38. Auditoría General de la Nación. Mallas de la alimentación [Internet]. Bogotá, Colombia; 2016. [citado 2016 Nov 26]. Disponible en: http: //www.auditoria.gov.co/ Analisis_Contratacion/MALLAS\%20EMPRESARIALES/Mallas_Alimentacion_Escolar.pdf

Artículo presentado en $01 / 11 / 2017$

Aprobado en 14/03/2019

Versión final presentada en 16/03/2019 